\title{
Multi-Objective Gust Load Alleviation Control Designs for an Aeroelastic Wind Tunnel Demonstration Wing
}

\author{
Michael Drew* \\ KBRwyle, Moffett Field, CA, 94035 \\ Kelley E. Hashemi ${ }^{\dagger}$ and Nick Cramer \\ NASA Ames Research Center, Moffett Field, CA, 94035 \\ Juntao Xiong ${ }^{\S}$ \\ KBRwyle, Moffett Field, CA, 94035 \\ Nhan T. Nguyen ${ }^{\text {II }}$ \\ NASA Ames Research Center, Moffett Field, CA, 94035
}

\begin{abstract}
This paper presents several control and gust disturbance estimation techniques applied to a mathematical model of a physical flexible wing wind tunnel model used in ongoing tests at the University of Washington Aeronautical Laboratory's Kirsten Wind Tunnel. Three methods of gust disturbance estimation are presented, followed by three control methods: LQG, Basic Multi-Objective (BMO), and a novel Multi-Objective Prediction Correction (MOPC) controller. The latter of which augments a multi-objective controller, and attempts to correct for errors in the disturbance estimate. A simplified linear simulation of the three controllers is performed and a simple MIMO stability and robustness assessment is performed. Then, the same controllers are simulated in a higher fidelity Simulink environment that captures sampling, saturation and noise effects. This preliminary analysis indicates that the BMO controller provides the best performance and largest stability margins.
\end{abstract}

\section{Introduction}

A $s$ aircraft manufacturers seek ways to reduce weight and increase efficiency by using lighter materials and higher aspect ratio wings, the resulting loss of structural rigidity may decrease aerodynamic performance, leading to increased drag, reduced flutter margins, and undesired loading. In recent years, researchers developed and matured Performance Adaptive Aeroelastic Wing (PAAW) technology, which incorporates novel control surfaces and control algorithms to actively shape the wing in order to mitigate these issues. One proposed technology, known as the Variable Camber Continuous Trailing Edge Flap (VCCTEF), uses many small multi-cambered control surfaces on each wing's trailing edge [1] to shape the span-wise lift distribution of the wing.

A collaboration among NASA, Scientific Systems Company, Inc. and Boeing Research and Technology led to further developments in the VCCTEF concept and included wind tunnel tests conducted at the University of Washington Aeronautical Laboratory's (UWAL) Kirsten Wind Tunnel. The first two test campaigns explored the VCCTEF's cruise $[2,3]$ and high-lift $[4,5]$ performance respectively. The third set of experiments conducted in December 2017 and June 2018 investigated the use of VCCTEF technology for real-time drag reduction [6]. With the addition of an upstream gust generator in the wind tunnel, a fourth set of experiments is slated for 2020 to explore the use of the VCCTEF for multi-objective control with gust load alleviation (GLA).

In the present study, the authors summarize several of the control and estimation strategies to be used for multiobjective control and GLA. Preliminary results are provided for the controllers via simulation on an aeroservoelastic (ASE) model of the physical wing used in the wind tunnel tests. Specifically, Section $\Pi$ contains a description of the ASE model used for the various control algorithms. Section III focuses primarily on two strategies of multi-objective

\footnotetext{
* Research Engineer, Intelligent Systems Division, AIAA Member

† Research Scientist, Intelligent Systems Division, AIAA Member, kelley.e.hashemi@nasa.gov

${ }^{\ddagger}$ Research Scientist, Intelligent Systems Division, AIAA Member

${ }^{\S}$ Research Engineer, Intelligent Systems Division, AIAA Member

"Technical Group Lead and Research Scientist, Intelligent Systems Division, AIAA Associate Fellow
} 


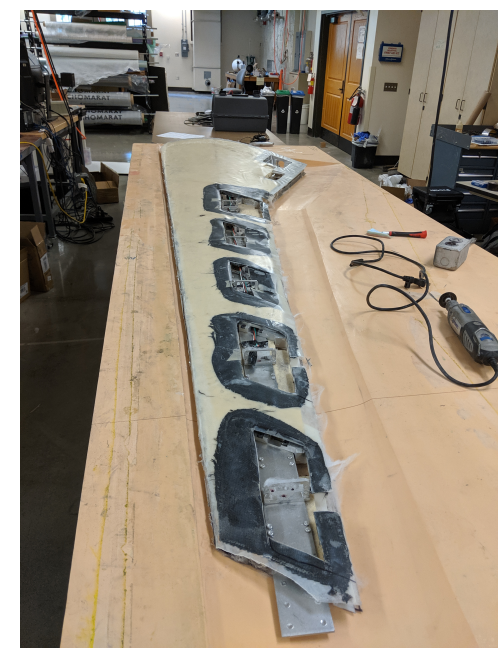

Fig. 1 Wing under construction

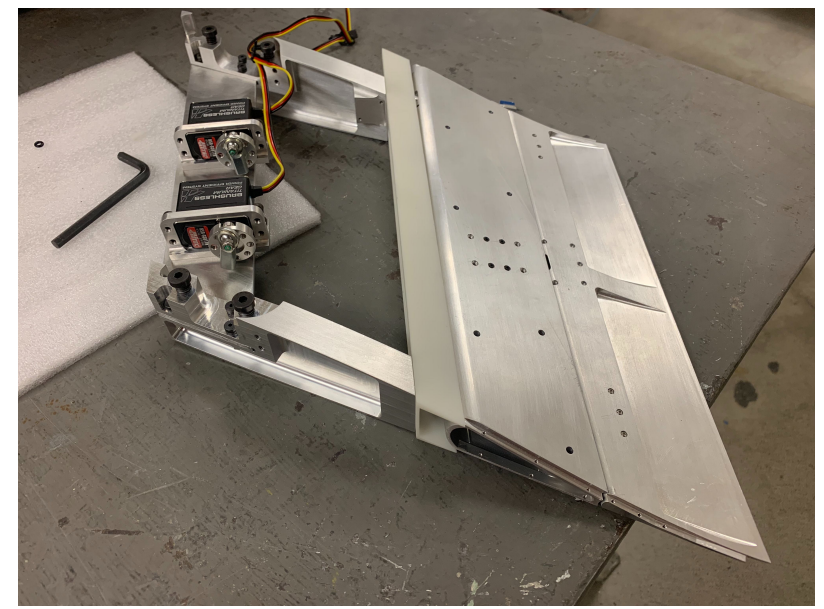

Fig. 2 VCCTEF flap under construction

control with GLA. Since an estimate of the gust disturbance is required for the GLA controller, Section IV]presents methods used in this work for estimating both the state vector and gust signal. Simulation results are presented and evaluated using some basic performance metrics in Section $\mathrm{V}$ along with a robustness assessment. Conclusions and an overview of this work's contributions and next steps are discussed in Section VI.

\section{The UWAL ASE Model}

A flexible wing based on the Common Research Model (CRM) geometry [7] was constructed to demonstrate active multi-objective GLA techniques. The $7 \mathrm{ft}$ half span wing includes VCCTEF actuation consisting of six individually actuated flaps and flexible inserts between flaps to provide a smooth trailing edge surface. Pictures of the wing and a flap during the construction process are provided in Fig. 1 and Fig. 2, respectively. Camber variation for the two inboard flaps is available through the use of a secondary hinge. Sensors used by the control designs include multiple accelerometers and strain gauges. A schematic of the sensing and actuation mechanisms is depicted in Fig. 3

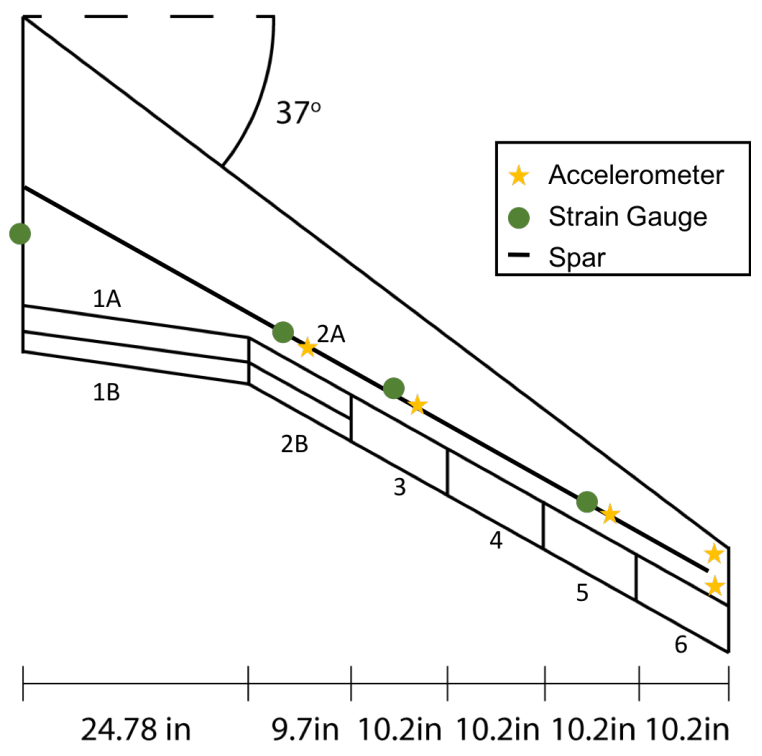

Fig. 3 Wing schematic indicating commandable flaps and sensor locations 


\section{A. Wing Model}

The mathematical representation of the UWAL ASE wing model is derived based on a sub-scale version of the CRM with the modified flap configuration previously indicated. The state space model is

$$
\begin{aligned}
& \dot{x}=A x+B_{n} u_{n}+B_{m} u_{m}+E d \\
& y=C x+D_{n} u_{n}+D_{m} u_{m}+F d
\end{aligned}
$$

where the $u_{n}$ control term is used to accommodate any nominal control laws that should be in place prior to multi-objective control action. For example, if a baseline drag minimizing configuration has been previously identified, $u_{n}$ can be used to command it. The $u_{m}$ control term is the multi-objective controller. It attempts to simultaneously provide:

1) Flexible motion stabilization

2) Gust and maneuver load alleviation

3) Drag minimization

The two control terms can utilize disparate control allocation.

The wing model has 9 control inputs consisting of angle of attack, $\alpha$, and the 8 control surfaces shown in Fig. 3 . Note that only some of the flaps are multi-cambered. Surfaces designated by $\mathbf{A}$ refer to the inner degree of freedom of a VCCTEF flap, and surfaces designated by $\mathbf{B}$ denote the trailing degree of freedom. Absence of a letter indicates traditional flap architecture. The mathematical model contains inputs for position, rate, and acceleration of each of these inputs, resulting in 39 inputs total. Outputs available in simulation include all six degrees of freedom (position, rate, and accelerations) for each of the 100 structural elements in the wing, plus the wing root forces and moments. These can be used to emulate IMU and strain gauge outputs with the addition of sensor dynamics and noise if desired. The gust disturbance applied to the model is a two-dimensional vector defined as $d=\left[\begin{array}{ll}w & \dot{w}\end{array}\right]^{T}$ where $w$ is the vertical gust rate.

The wing model states are defined as

$$
\boldsymbol{x}=\left[\begin{array}{cc}
\text { Mode Disp. } & q \\
\text { Mode Lag } & y \\
\text { Mode Lag } & z \\
\text { Mode Rate } & \dot{q} \\
\text { Mode Rate Lag } & \dot{y} \\
\text { Mode Rate Lag } & \dot{z} \\
\text { Flap Lag } & v \\
\text { Flap Lag } & w \\
\text { Flap Lag Rate } & \dot{v} \\
\text { Flap Lag Rate } & \dot{w} \\
\text { Gust Lag } & x_{g} \\
\text { Gust Lag Rate } & \dot{x}_{g}
\end{array}\right] .
$$

Note that, since this is a wing-only model, there are no rigid body states. The number of structural modes present in the model $n_{\text {modes }}$ depends on the desired level of fidelity. There are $6 n_{\text {modes }}$ structural states consisting of the generalized displacements and rates, plus lag states arising from the R.T. Jones approximation. There are also 32 flap lag states and their rates for the 8 controllable flap joints. With 20 modes present, and 2 gust lag states, the resulting model has 154 states. A thorough derivation of this model can be found in Ref. 8 .

Relevant performance metrics for the wing model include wing root bending moment

$$
M_{y}=M_{x} x+M_{u_{n}} u_{n}+M_{u_{m}} u_{m}+M_{w} d .
$$

Additionally, the change in drag from a drag-optimized baseline configuration is

$$
\begin{aligned}
\Delta C_{D}=C_{D_{x}} x+C_{D_{u_{n}}} u_{n}+C_{D_{u_{m}}} u_{m}+x^{T} C_{D_{x^{2}} x}+x^{T} C_{D_{x n}} u_{n}+x^{T} C_{D_{x m}} u_{m} \\
+u_{n}{ }^{T} C_{D_{u^{2} n n}} u_{n}+u_{m}{ }^{T} C_{D_{u^{2} m m}} u_{m}+u_{n}^{T}\left(C_{D_{n m}}+C_{D_{m n}}^{T}\right) u_{m} .
\end{aligned}
$$

Note that $\Delta C_{D}$ will always be positive due to the choice of baseline. 


\section{B. Actuator Model}

The 8 flaps are modeled using a simple second-order model based on the Dynamixel W350R step response. For the angle of attack input, slow second-order angle of attack dynamics are used. Since this input is typically used as the nominal control input, its dynamics are considerably less important than the flap dynamics. Combining the angle of attack dynamics along with the 8 identical flap dynamics, the state space actuator model can be represented as

$$
\begin{aligned}
\dot{x}_{a} & =A_{a} x_{a}+B_{a_{n}} u_{n_{c}}+B_{a_{m}} u_{m_{c}} \\
u_{n} & =C_{a_{n}} x_{a}+D_{a_{n}} u_{n_{c}} \\
u_{m} & =C_{a_{m}} x_{a}+D_{a_{m}} u_{m_{c}}
\end{aligned}
$$

Here, $u_{n_{c}}$ and $u_{m_{c}}$ are the commanded deflections. The resulting nominal and multi-objective inputs $u_{n}$ and $u_{m}$ include actual deflection, rate, and acceleration inputs for the wing model. Note that actuator dynamics are neglected for control gain calculation in this study.

\section{Controller Design}

The model accommodates two separate control laws: the nominal controller, $u_{n}$, and the multi-objective controller, $u_{m}$. While this paper focuses on the design and performance of $u_{m}$, the structures of both are reviewed in this section and both will be used in simulation.

\section{A. Nominal Control}

If there exists a nominal controller to be used, it is assumed to have the form

$$
u_{n}=K_{n x} x+K_{n r} r+K_{n w} d+\Lambda_{n}
$$

where terms can be zeroed as necessary if unused. Design of any nominal controller is taken to be outside the scope of this paper. The nominal-loop-closed dynamics are then

$$
\dot{x}=\bar{A} x+\bar{B}_{i} r+\bar{\Lambda}_{n}+B_{m} u_{m}+\bar{E} d
$$

where state can be augmented with integrator states as necessary to accommodate servomechanism tracking of commands $r$. The associated performance metrics are

$$
\begin{aligned}
& M_{y}=\bar{M}_{x} x+\bar{M}_{r} r+M_{u_{m}} u_{m}+\bar{M}_{w} d+\bar{\Lambda}_{M} \\
& \Delta C_{D}=\bar{C}_{D_{x}} x+\bar{C}_{D_{r}} r+\bar{C}_{D_{w}} d+\bar{C}_{D_{u_{m}}} u_{m}+\bar{\Lambda}_{C}+x^{T} \bar{C}_{D_{x^{2}}} x+u_{m}^{T} \bar{C}_{D_{u^{2} m m}} u_{m}+x^{T} \bar{C}_{D_{x u_{m}}} u_{m}+r^{T} \bar{C}_{D_{r u_{m}}} u_{m} \\
& +d^{T} \bar{C}_{D_{w u_{m}}} u_{m}+r^{T} \bar{C}_{D_{r x}} x+d^{T} \bar{C}_{D_{w x}} x+r^{T} \bar{C}_{D_{r^{2}}} r+r^{T} \bar{C}_{D_{r w}} d+d^{T} \bar{C}_{D_{w^{2}}} d .
\end{aligned}
$$

Matrix definitions are given in Appendix A.

In the present study, the nominal control is restricted to an open loop angle of attack setting. This design choice was predicated on the design and configuration of the physical wind tunnel wing model, where angle of attack is typically known and set prior to an experiment. Thus, the nominal control law used in subsequent simulations is simply

$$
u_{n}=r=\alpha_{c}
$$

where $r$ is the commanded angle of attack $\alpha_{c}$.

\section{B. Multi-Objective Control}

The command of the flaps to reduce structural motion, gust/maneuver load, and drag are herein generically denoted "multi-objective" control. In this paper we define and investigate three controller designs for $u_{m}$ intended to achieve at least some subset of these goals. 


\section{1. $L Q R / G$ Controller}

Used as a baseline for comparison, this is the standard LQR/G control law. LQR results may be investigated but only as a purely academic exercise, since plant internal states cannot, in practice, be known. In simulation, LQR is only feasible when the truth model and control model are identically sized. Thus, for this work the baseline control law is an LQG controller defined as

$$
u_{m}=K_{x} \hat{x}
$$

where state estimates $\hat{x}$ are produced by a fixed-gain (infinite horizon) Kalman optimal observer. Note that this controller is not, strictly speaking, "multi-objective" since it is only capable of suppressing structural motion by regulating the wing's structural mode states to zero. There is no knowledge of gust disturbance estimates, nor are there means to explicitly tune the controller for wing root bending moment or drag reduction.

\section{Basic Multi-Objective Controller}

A multi-objective optimal control design that attempts to address all goals is obtained through minimization of the cost function

$$
J=\lim _{t_{f} \rightarrow \infty} \frac{1}{2} \int_{0}^{t f}\left(x^{T} Q x+u_{m}^{T} R_{m} u_{m}+q_{M} M_{y}^{2}+q_{D} \Delta C_{D}\right) d t
$$

subject to the dynamic constraint in Eq. (7). $Q, R_{m}, q_{M}$, are $q_{D}$ are appropriately-sized user-selected weights. The resulting controller has the form

$$
u_{m}=K_{x} \hat{x}+K_{r} r+K_{w} \hat{d}+\Lambda .
$$

The derivation of this control law is discussed in greater detail in [11] and gain definitions can be found in Appendix B. Note that an observer must be used to obtain an estimate of the state, $\hat{x}$, for implementation of $u_{m}$. More importantly, an estimate of the gust, $\hat{d}$, must also be obtained and two novel procedures are later considered in Section IV.B

\section{Multi-Objective + Prediction Correction Controller}

The last option considers an additional control term, $u_{c}$, for the purpose of estimated quantity error correction

$$
\dot{x}=A x+B_{n} u_{n}+B_{m}\left(u_{m}+u_{c}\right)+E d .
$$

To determine $u_{c}$, the $u_{n}$ and basic multi-objective $u_{m}$ loops are closed and a cost function that seeks to minimize the error $e=x-x_{m}$ is used. Here, $x$ captures the true dynamics being driven by the true gust, while a best-known model of those dynamics being driven by an estimate of the gust are captured by $x_{m}$. The appropriate cost function is given by

$$
J=\lim _{t_{f} \rightarrow \infty} \frac{1}{2} \int_{0}^{t f}\left(e^{T} Q_{c} e+u_{c}^{T} R_{c} u_{c}+q_{c M} \tilde{M}_{y}^{2}+q_{c D} \Delta \tilde{C}_{D}^{2}\right) d t
$$

where the $\tilde{M}_{y}$ and $\Delta \tilde{C}_{D}$ terms are included to prevent washout of performance benefits with use of $u_{c}$. The error signal's true and modeled dynamics are given by

$$
\begin{gathered}
\dot{x}=\overline{\bar{A}} x+\overline{\bar{B}}_{i} r+\overline{\bar{\Lambda}}_{n}+B_{m} u_{c}+\overline{\bar{E}} d \\
\dot{x}_{m}=\overline{\bar{A}} x_{m}+\overline{\bar{B}}_{i} r+\overline{\bar{\Lambda}}_{n}+\overline{\bar{E}} \hat{d}
\end{gathered}
$$

such that the dynamics for the error that constrain the cost function are

$$
\dot{e}=\overline{\bar{A}} e+B_{c} u_{c}+\overline{\bar{E}} \tilde{d} .
$$

Matrix definitions are again given in Appendix A. Note that $\tilde{d}=d-\hat{d}$. Similarly, $\tilde{M}_{y}$ and $\Delta \tilde{C}_{D}$ are formed using the $x_{m}$ dynamics according to

$$
\begin{aligned}
\tilde{M}_{y} & =M_{y}-M_{y_{m}} \\
& =\left[M_{x} x+M_{u_{n}} u_{n}+M_{u_{m}}\left(u_{m}+u_{c}\right)+M_{w} d\right]-\left[M_{x} x_{m}+M_{u_{n}} u_{n}+M_{u_{m}} u_{m}+M_{w} \hat{d}\right] \\
& =M_{x} e+M_{u_{m}} u_{c}+M_{w} \tilde{d}
\end{aligned}
$$




$$
\begin{aligned}
\Delta \tilde{C}_{D} & =\Delta C_{D}-\Delta C_{D_{m}} \\
& =\left[C_{D_{x}} x+C_{D_{u n}} u_{n}+C_{D_{u m}}\left(u_{m}+u_{c}\right)\right]-\left[C_{D_{x}} x_{m}+C_{D_{u n}} u_{n}+C_{D_{u m}} u_{m}\right] \\
& =C_{D_{x}} e+C_{D_{u m}} u_{c}
\end{aligned}
$$

Note that only the linear portion of the $\Delta C_{D}$ expression is utilized for tractability.

The resulting controller has the form

$$
u_{c}=\bar{K}_{e} e+\bar{K}_{w} \tilde{d}
$$

with gain definitions given in Appendix B.

With the true disturbance unknown, the disturbance estimation error $\tilde{d}$ needs to be approximated. This can be done based on selected plant and model outputs $y$ and $y_{m}$ defined as

$$
\begin{aligned}
y & =\overline{\bar{C}} x+\overline{\bar{D}}_{i} r+D_{m} u_{c}+\overline{\bar{F}} d+\overline{\bar{\Lambda}}_{y} \\
y_{m} & =\overline{\bar{C}} x_{m}+\overline{\bar{D}}_{i} r+\overline{\bar{F}} \hat{d}+\overline{\bar{\Lambda}}_{y} .
\end{aligned}
$$

Depending on the choice and number of outputs, $\tilde{d}$ can be calculated as

$$
\tilde{d}=\left(\overline{\bar{F}}+D_{c} \bar{K}_{w}\right)^{-1}\left[\left(y-y_{m}\right)-\left(\overline{\bar{C}}+D_{c} \bar{K}_{e}\right) e\right]
$$

with the pseudoinverse being necessary depending on the dimensions of $y$ and $d$.

\section{State and Disturbance Estimation}

State estimates $\hat{x}$ are determined using the standard Kalman state estimator technique with fixed gains calculated using the $A$ and $C$ matrices of the wing plant model. Actuator dynamics and their states $x_{a}$ are neglected, but gust estimates may be provided to the estimator if desired. The model is found to not be strictly observable, but since it is open-loop stable, an appropriate choice of outputs can result in a detectable $(A, C)$ pair. As Section $\nabla$ demonstrates, it is found that a reduced state space model may be used for state estimation and feedback control gain calculations, however, model reduction is not necessary to calculate usable observer gains.

Though state estimation is not a challenge with this model, disturbance gust estimation can be problematic. The following subsections discuss the three approaches that have been explored.

\section{A. Extended State and Gust Estimator}

There are several known methods for estimating system disturbances, and in particular gust disturbance. Hashemi et al. in Ref. 11 describes several gust estimation strategies in the context of the Generic Transport Model (GTM). These include estimating the gust system matrices, adaptive gust estimation, and gust estimation using an extended state estimator. In Ref. 10, the extended state and gust estimator is used for a full scale CRM model with aspect ratio 13.5.

Since the gust signal disturbance consists of vertical velocity and acceleration $\left[\begin{array}{ll}w & \dot{w}\end{array}\right]^{T}$, the extended state and gust estimator takes the form

$$
\begin{aligned}
& \frac{d}{d t}\left[\hat{d}=\left[\begin{array}{c}
\hat{x} \\
\hat{w} \\
\hat{\dot{w}}
\end{array}\right]\right]=\left[\begin{array}{ccc}
A & \multicolumn{2}{c}{E} \\
0 & 0 & 1 \\
0 & 0 & 0
\end{array}\right]\left[\begin{array}{c}
\hat{x} \\
\hat{d}
\end{array}\right]+\left[\begin{array}{c}
B_{n} \\
0 \\
0
\end{array}\right] u_{n}+\left[\begin{array}{c}
B_{m} \\
0 \\
0
\end{array}\right] u_{m}+L(y-\hat{y}) \\
& \hat{y}=\left[\begin{array}{ll}
C & F
\end{array}\right]\left[\begin{array}{l}
\hat{x} \\
\hat{d}
\end{array}\right]+D_{n} u_{n}+D_{m} u_{m} .
\end{aligned}
$$

Thus, both state and gust estimates are achieved simultaneously. Note that the state estimate $\hat{x}$ from above may actually be a reduced state vector if model reduction is employed.

While this estimator has been shown to provide accurate estimates of both states and gust disturbances, tuning the gains can be difficult. The resulting closed loop system typically features stiff dynamics that can be difficult to integrate in simulation, and tend to reduce loop stability margins. Thus, alternative estimation methods are explored. 


\section{B. Gust Vane Transfer Function}

The ultimate goal of this work is to utilize look-ahead sensor technologies like LIDAR to estimate gust for use in GLA control. Absent this type of sensing within the confines of a wind tunnel experiment, another option is to use the known signal applied to the gust generator (shown in Fig. 4) and convert it into an equivalent disturbance vector $d=\left[\begin{array}{cc}w & \dot{w}\end{array}\right]^{T}$. To this end, Xiong et al. in Ref. 12 performed CFD analysis for the gust generator geometry as depicted

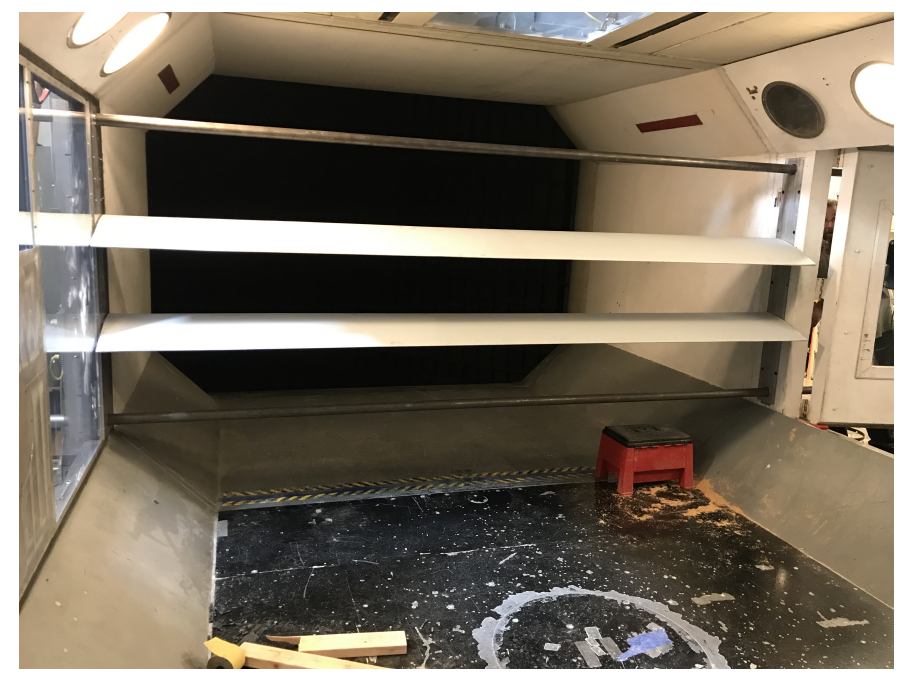

Fig. 4 UWAL gust generator installation

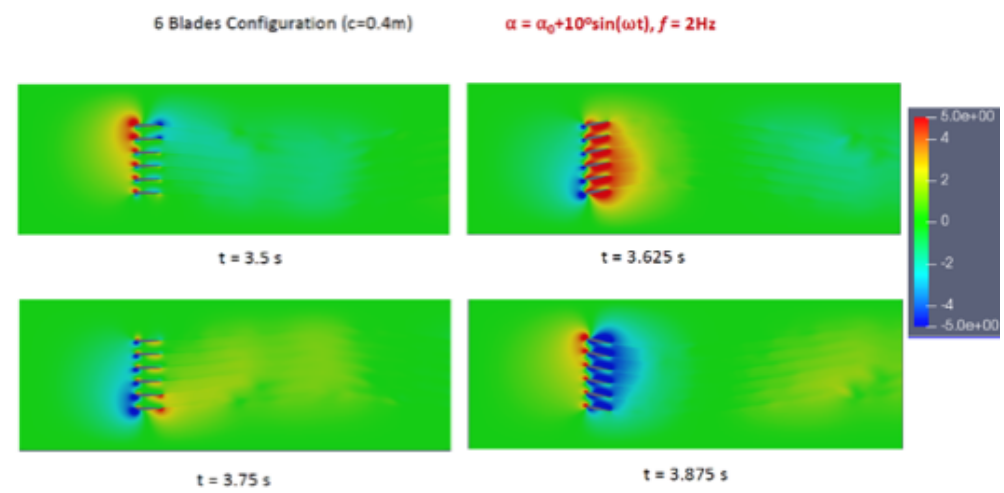

Fig. 5 CFD analysis of UWAL gust generator geometry

in Fig. 5. The results were used to characterize the gust signal at the wing model's leading edge resulting from a sinusoidal commanded vane angle of $\theta_{v_{c}}= \pm 10^{\circ}$ at $1 \mathrm{~Hz}$. A second-order linear transfer function plus transport delay is fit to the CFD results and used for estimating the gust disturbance with

$$
\frac{\hat{w}(s)}{\theta_{v_{c}}(s)}=e^{-0.06 s} \frac{0.2006 s+2.198}{s+6.8} .
$$

This method produces good results for a specific signal frequency and amplitude in simulation as shown in Fig.6 However, it is predicated on a specific signal generated from a CFD simulation, which may fail to capture real-world flow behavior within the tunnel. Even the CFD results demonstrate that the relationship between $\theta_{v_{c}}$ and $\hat{w}$ is nonlinear with respect to amplitude and frequency. Thus, the above transfer function is not likely to be very accurate when applied in the wind tunnel without being re-fit so that it can be used during real-time gust estimation. 


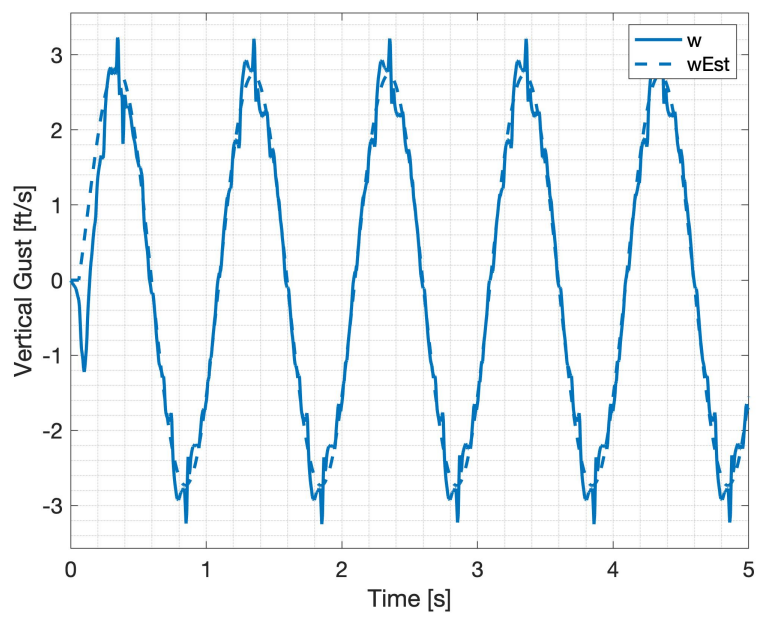

Fig. 6 Simulated gust disturbance estimation using the gust vane transfer function

\section{Reactive Gust Estimation}

The final method of estimating gust uses a dynamic update law given by

$$
\dot{\hat{d}}=-\Gamma\left(v \hat{d}-F_{g}^{-1}\left(y_{g}-\hat{y}_{g}\right)\right)
$$

where $\cdot_{g}$ refers to quantities associated with outputs used for gust estimation. The estimated output is given by

$$
\hat{y}_{g}=C_{g} \hat{x}+D_{n_{g}} u_{n}+D_{m_{g}} u_{m}+F_{g} \hat{d},
$$

and $\Gamma$ and $v$ are tunable parameters. $F_{g}^{-1}$ may require a pseudoinverse depending on the dimensions of $y_{g}$ and $d$.

This approach is dependent on the model accuracy and can be sensitive to the tuning parameters, often producing noisy results. However, it is BIBO stable and computationally simple to compute in simulation or real time.

\section{Results}

A simulation of the entire UWAL CRM ASE model is built within the Simulink environment, and is integrated using a fixed time step Bogacki-Shampine ODE solver. It contains the wing model described above, actuator dynamics (with saturation logic included), state and gust estimators, and controllers. The choice of estimator and controller is selected at run time. In order to capture some of the real world experimental timing issues, the model contains sampling zero-order hold blocks for both the actuators and sensors. Also, noise and filtering can be added on the sensor side to mimic accelerometer and strain gauge behavior. The top level view of this model is shown in Fig.77

In parallel with the Simulink model, a linear version can be run directly using MATLAB's "lsim" function. The linear version does not capture sampling, saturation effects, or noise, but runs much faster, and is useful for quick controller tuning and for analyzing closed loop stability margins. Comparing the performance of the two models also aids in verifying the model's accuracy.

The following subsections compare the performance and stability margins of the LQG, Basic Multi-Objective Controller (BMO), and the Multi-Objective + Prediction Correction Controller (MOPC). As mentioned earlier, the nominal control law consists of an open loop setting for angle of attack. The simulation results presented herein use $\alpha_{c}=0$. Since the physical wing model shown in Fig. 3 does not use multi-cambered flaps for positions 3-6, the multi-objective control allocation consists of flaps 1B, 2B, 3, 4, 5, 6 (1A and 2A are unused). The "truth" model used in all simulations is the full 154 state model described above with additional actuator dynamics also included. As is typical for computational efficiency, the model used for control and estimation is substantially reduced depending on the controller. For the BMO and MOPC controllers, which require a gust estimate, the gust vane transfer function method is used. The state estimator uses two plant outputs: wing tip vertical acceleration, and wing root bending moment. The gust estimate is also utilized by the state estimator. Finally, since the main thrust of this work concerns gust load alleviation, and since an accurate drag model for the wing has yet to be created, we focus on wing root bending moment reduction rather than drag reduction. 


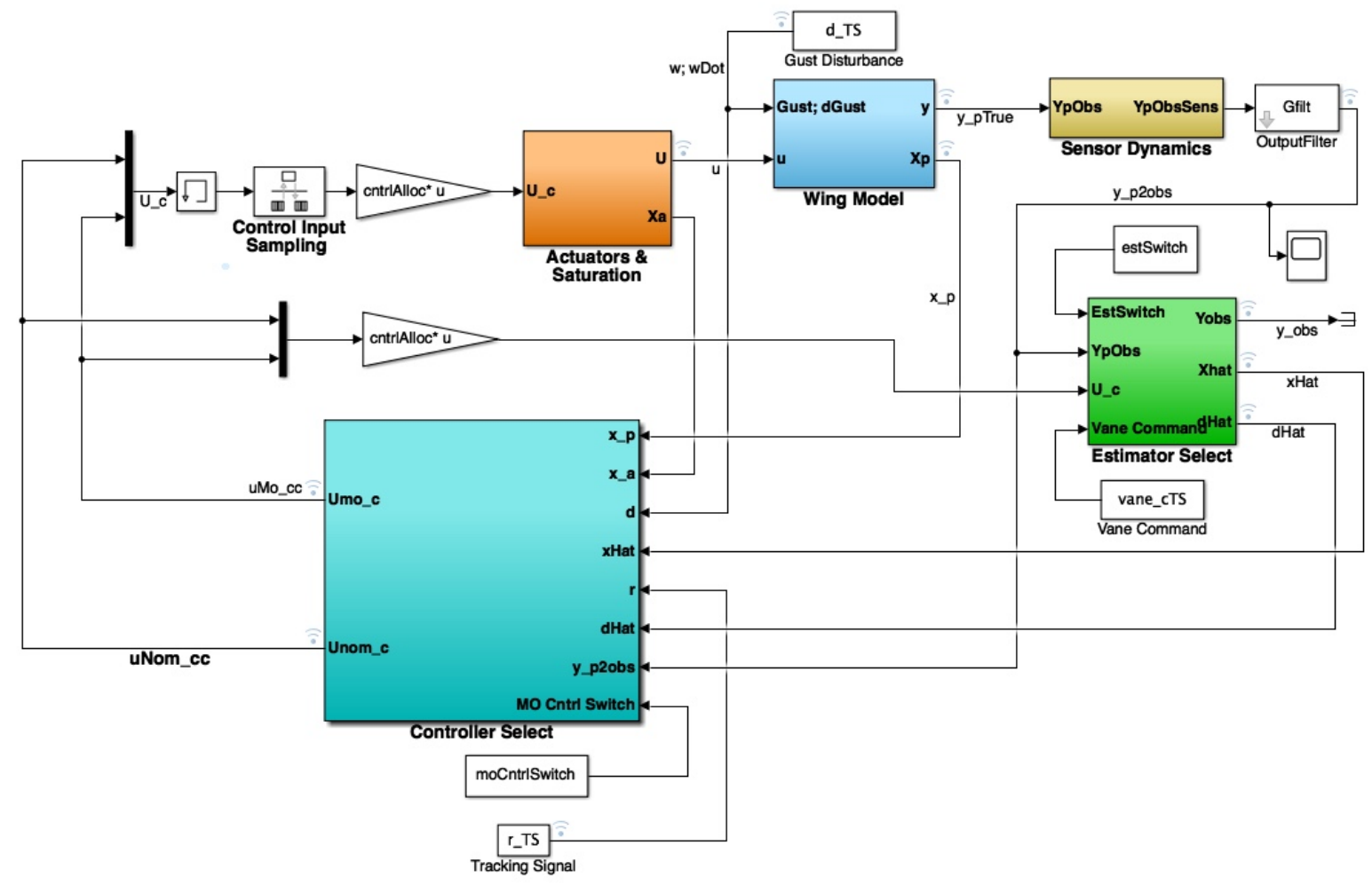

Fig. 7 Simulink model of the UWAL CRM ASE simulation

\section{A. Linear MatLab Simulation}

First, linear simulations are conducted in MATLAB and tuned such that flap deflection does not substantially exceed $\pm 15^{\circ}$. The linear system models are constructed with analysis points at appropriate plant inputs and outputs for a subsequent MIMO stability assessment. The wing model's first 5 structural modes are at 2.8,11.3,13.2, 27, and 33 Hz. Therefore, given the physical actuator rate limits, and the low probability of exciting higher modes in the wind tunnel, the model used for control gains and estimation is reduced using the singular perturbation method. The first two controllers (LQG and BMO) use a reduced model with 56 states for control gain and estimation calculations consisting of only the first bending mode and flap aeroelastic lag states. The MOPC controller utilizes a 62 state model, which captures the first 2 modes. None of the simulations consider actuator dynamics. Again, all simulations $d o$ use the full model plus actuators as the "true" plant.

\section{Linear Simulation Results}

Figure 8 shows the performance results of the three controllers relative to the open loop behavior, and Fig. 9 shows the resulting flap deflections. From a qualitative standpoint, it is easy to observe in Fig. 8a that both LQG and BMO reduce the first mode response similarly, with the BMO controller featuring a smoother flap response. Since the results of Fig. $8 \mathrm{~b}$ are difficult to judge, Table 1 shows the root mean square of wing root bending moment, and its normalized value relative to the gust disturbance.

The MOPC controller was difficult to tune without loosing stability. The results show only a marginal improvement over the open loop behavior, with an erratic flap response. It is not yet clear if additional tuning or structural changes to the correction design would improve the stability and performance of this controller, but the theoretical merits of the design suggest that performance gains should be available when properly applied.

\section{Stability Margin Analysis}

The above results show that in terms of wing root bending moment and structural mode suppression, the BMO controller does not offer a significant advantage over standard LQG control. However, a distinction is found by 


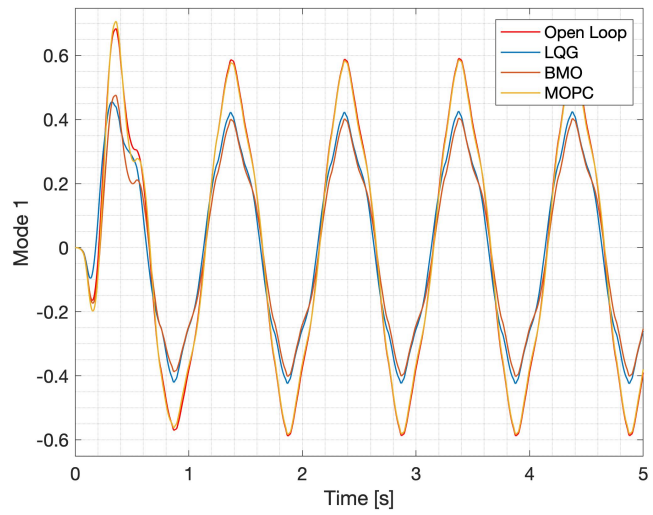

(a) Generalized mode 1 displacement state

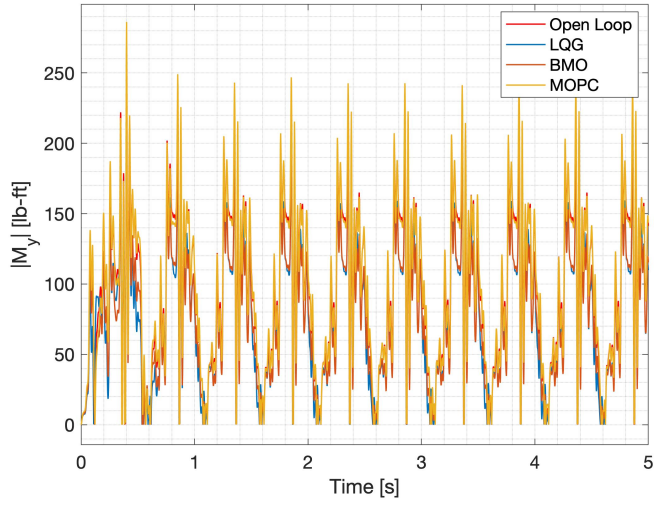

(b) Absolute value of wing root bending moment $\left|M_{y}\right|$

Fig. 8 Linear simulation results showing modal displacement and wing root bending moment.
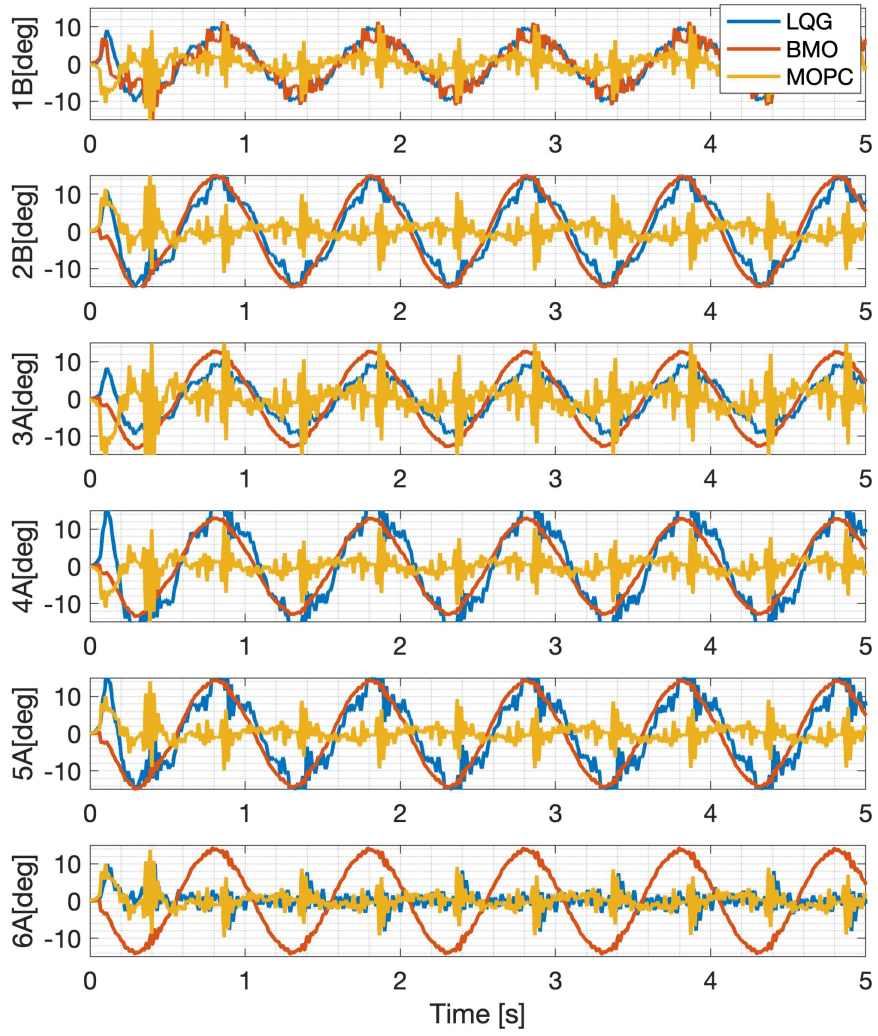

Fig. 9 Linear simulation controller flap deflections

performing a stability and robustness assessment of the three controllers.

This analysis is done by individually breaking the control loop at the multi-objective control inputs and the plant outputs at the points indicated by the green dots in Fig. 10 Then, a margin analysis on the resulting transfer functions is performed. For instance, breaking the loop at the input as shown in Fig. 11 would result in the relationship $u_{\text {in }}$ out $=-L_{\text {in }}(s) u_{\text {in }}$. 


\begin{tabular}{|l|c|c|}
\hline & $\mathbf{R M S}\left(M_{y}\right)$ & $\mathbf{R M S}\left(M_{y}\right) / \mathbf{R M S}($ gust $)$ \\
\hline Open Loop & 113.9 & 59.23 \\
\hline LQG & 91.94 & 47.81 \\
\hline BMO & 90.12 & 46.86 \\
\hline MOPC & 112.8 & 58.66 \\
\hline
\end{tabular}

Table 1 Controller performance metrics from linear simulation

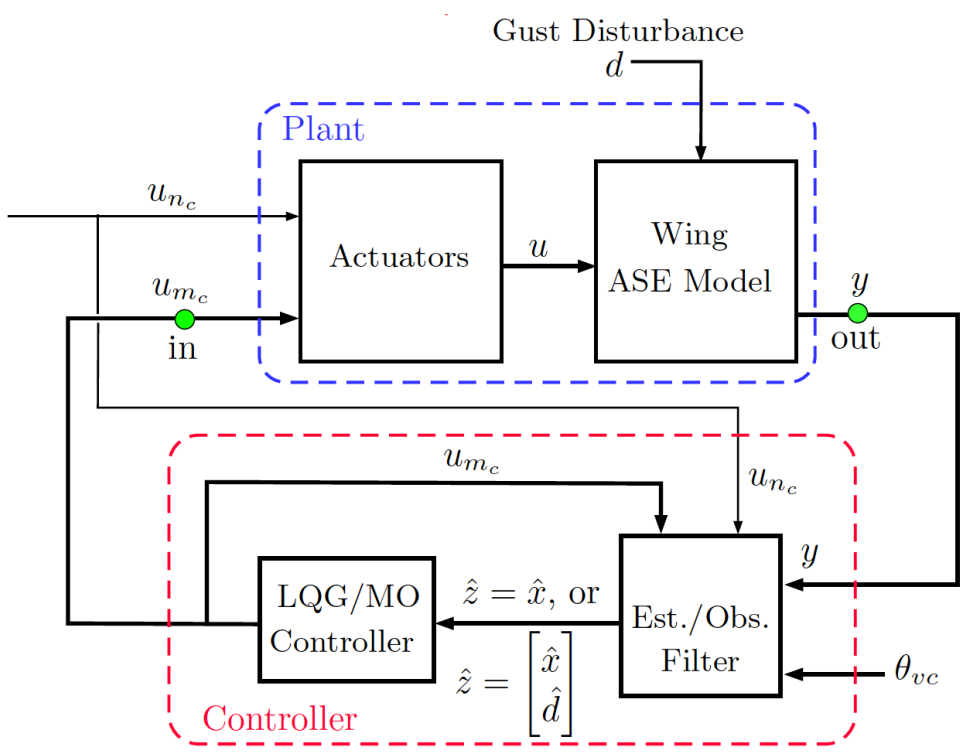

Fig. 10 Schematic depiction of the UWAL CRM ASE control model

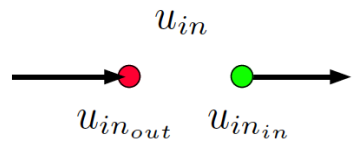

Fig. 11 Input loop gain break with $u_{\text {out }}=-L_{\text {in }}(s) u_{\text {in }}$

$L_{i n}(s)$ is extracted from the system using MATLAB's analysis points feature, from which both the return difference and stability robustness transfer functions are obtained. Since $L_{i n}(s)$ is a $6 \times 6$ transfer function, a conservative approximation of the MIMO gain and phase margins at the plant input is calculated using a minimum singular value decomposition frequency analysis on both these transfer functions. This technique is discussed in 13. The output loop analysis is conducted by individually breaking the output loops, and analyzing the resulting SISO transfer functions in the traditional manner.

The Bode plots of the two plant output loop transfer functions are shown in Fig. 12, and a summary of the input and output loop margins is shown in Table 2. These results demonstrate that while the BMO controller performs similarly to the LQG controller, it does so with significantly improved margins because it partially relies on a stable disturbance estimate to drive control. The MOPC controller also features good margins, but its performance suffered due to high tuning sensitivities and stability issues discussed above.

Finally In Fig. 13 , the maximum singular value of the controller transfer function $G_{c}(s)$, which includes the estimator, control gains, and model dynamics (MOPC), is plotted for the three controllers. This plot shows that the BMO controller does a better job than LQG of attenuating sensor noise that may be passed to the plant. This is because, unlike the LQG controller that achieves its performance using higher state weights, the BMO controller relies more on the $\bar{K}_{w}$ term of Eq. 21, and utilizes the gust estimate as a feedforward term. The MOPC controller may have problems with stability at higher frequencies. 


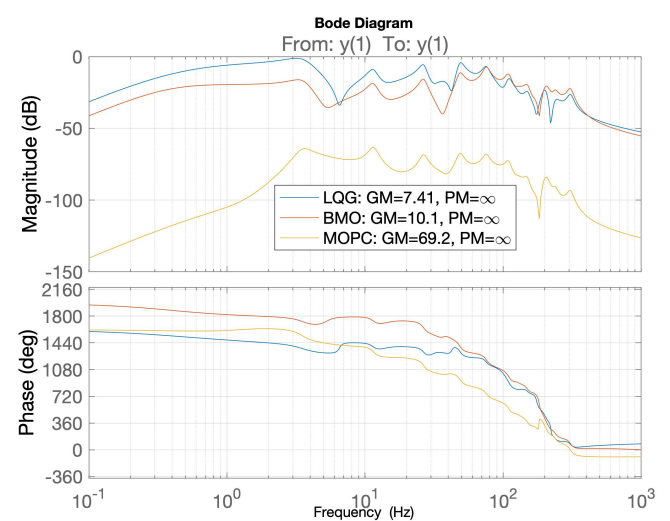

(a) Wing tip accelerometer output: $L_{o u t_{1}}(s)$.

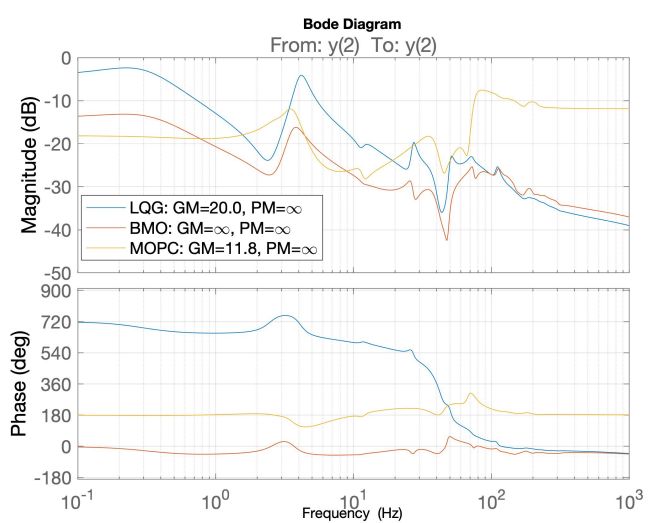

(b) Wing root bending moment output: $L_{\text {out }_{2}}(s)$.

Fig. 12 Loop gain analysis at the plant outputs

\begin{tabular}{|l|c|c|c|c|c|c|}
\cline { 2 - 7 } \multicolumn{1}{c|}{} & \multicolumn{2}{c|}{ Input MIMO Analysis } & \multicolumn{2}{c|}{ Output 1 } & \multicolumn{2}{c|}{ Output 2 } \\
\hline & GM [dB] & PM [deg] & GM [dB] & PM [deg] & GM [dB] & PM [deg] \\
\hline LQG & 3.30 & 18.20 & 7.41 & $\infty$ & 20.00 & $\infty$ \\
\hline BMO & 8.32 & 35.89 & 10.10 & $\infty$ & $\infty$ & $\infty$ \\
\hline MOPC & 5.84 & 28.36 & 69.20 & $\infty$ & 11.80 & $\infty$ \\
\hline
\end{tabular}

Table 2 Controller stability margin comparison

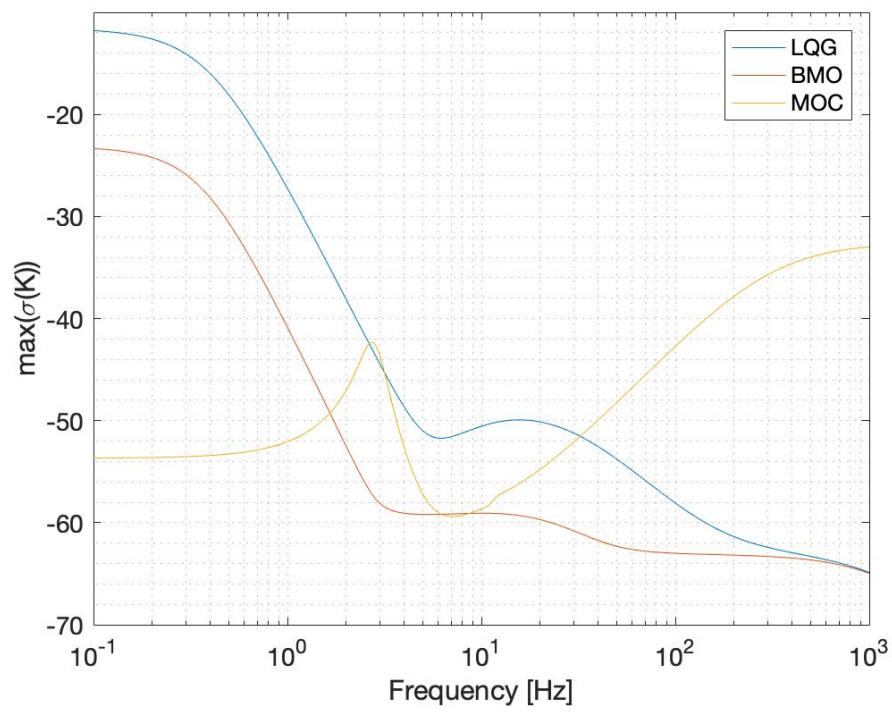

Fig. 13 Controller response $u_{m_{c}}=G_{c}(s) y(s)$.

\section{B. Simulink Simulation}

Next, in order to test the controllers on a more realistic model, they are run in the Simulink model shown in Fig.7 Noise is added to the accelerometer signal according to its data sheet. The sampling rates for the wing root bending moment (which originates from the wind tunnel's side-wall balance sensor) and the accelerometers are set to 50 and 80 $\mathrm{Hz}$ respectively, and the actuator sampling rate is set to $100 \mathrm{~Hz}$. These rates are implemented as zero-order-hold blocks in Simulink, and they are based on an estimate of the hardware as it exists in the design phase at the time of this writing. 
They will likely change once implemented, but not substantially. Computational delay can be enforced as a transport delay at the control output, but it is not yet known, and not applied during these simulations. Flap deflection and rate limits are enforced to $\pm 15^{\circ}$, and $\pm 45^{\circ} / \mathrm{s}$. These limits are an optimistic estimate of the actual servo and flap design performance capabilities.

The results of running the three controllers (tuned identically to the linear simulation above) in the Simulink model are shown in Figs. 14 and 15. Similar to the linear simulation, the LQG and BOM controllers suppress the first bending mode motion the best. Flap deflections shown in Fig. 15 reveal that all three controllers saturate the flaps in terms of both deflection and rate. Here, commanded deflection is shown with dashed lines, and actual are solid. Again, the MOPC controller shows the most erratic behavior in terms of flap deflection, but its actual saturation (calculated as $\left.\left|\delta_{c}-\delta\right|\right)$ is less than that of the other controllers. Table 3 summarizes several performance metrics. Similar to the linear simulation from the previous section, the BMO controller exhibits the best performance. Of course, performance is generally worse than in the linear simulation due to saturation, noise, and timing effects, however, none of these effects exceed the stability margins of the controllers.

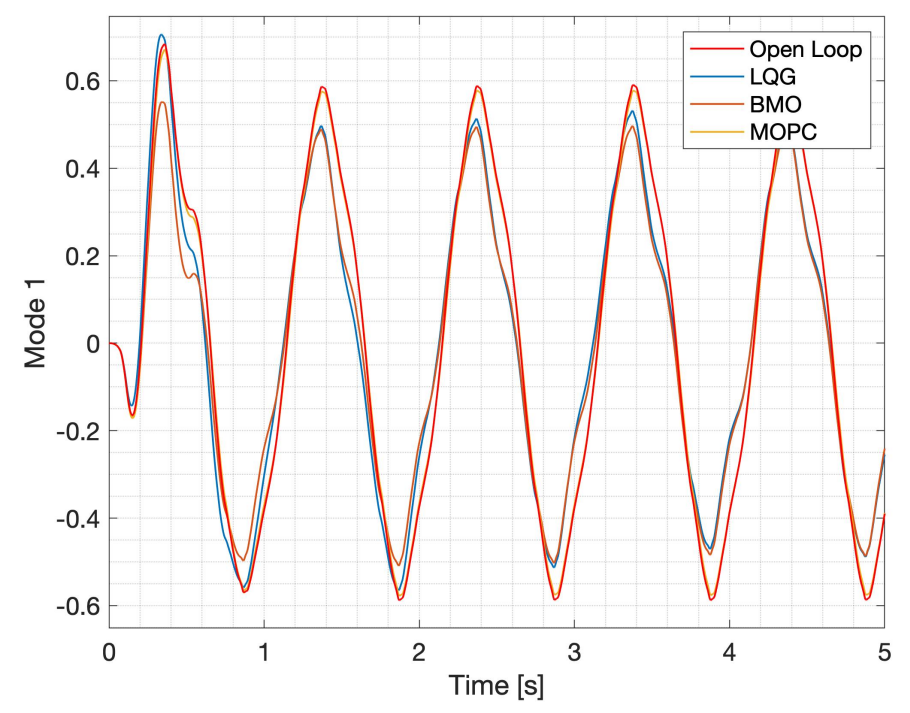

Fig. 14 Generalized mode 1 displacement state results from the Simulink model.

\begin{tabular}{|l|c|c|c|c|}
\hline & $\mathbf{R M S}\left(M_{y}\right)$ & $\mathbf{R M S}\left(M_{y}\right) / \mathbf{R M S}($ gust $)$ & Mean $\left|\delta_{c}\right|[\mathbf{d e g}]$ & Mean $\left|\delta_{c}-\delta\right|[\mathbf{d e g}]$ \\
\hline LQG & 102.57 & 53.33 & 8.93 & 6.69 \\
\hline BMO & 99.90 & 51.94 & 8.21 & 5.03 \\
\hline MOPC & 111.08 & 57.76 & 2.62 & 2.02 \\
\hline
\end{tabular}

Table 3 Controller performance metrics from Simulink simulation

These results indicate that flap saturation caused by limitations of the experimental hardware will likely curtail the potential of the multi-objective controllers. Therefore, it may be necessary to reduce the intensity and frequency of the gust during wind tunnel experiments in order to demonstrate the controller's potential.

\section{Conclusions}

Research continues to investigate the innovations that Performance Adaptive Aeroelastic Wing (PAAW) technology can provide for achieving several objectives simultaneously. This paper has presented several gust estimation techniques, in addition to a comparison of controllers designed to reduce structural motion, loading and ultimately, drag-especially in the presence of gust disturbances. It has been demonstrated that by using an accurate estimate of the gust signal, a more robust controller can be implemented with equal or better performance and stability margins than the conventional 

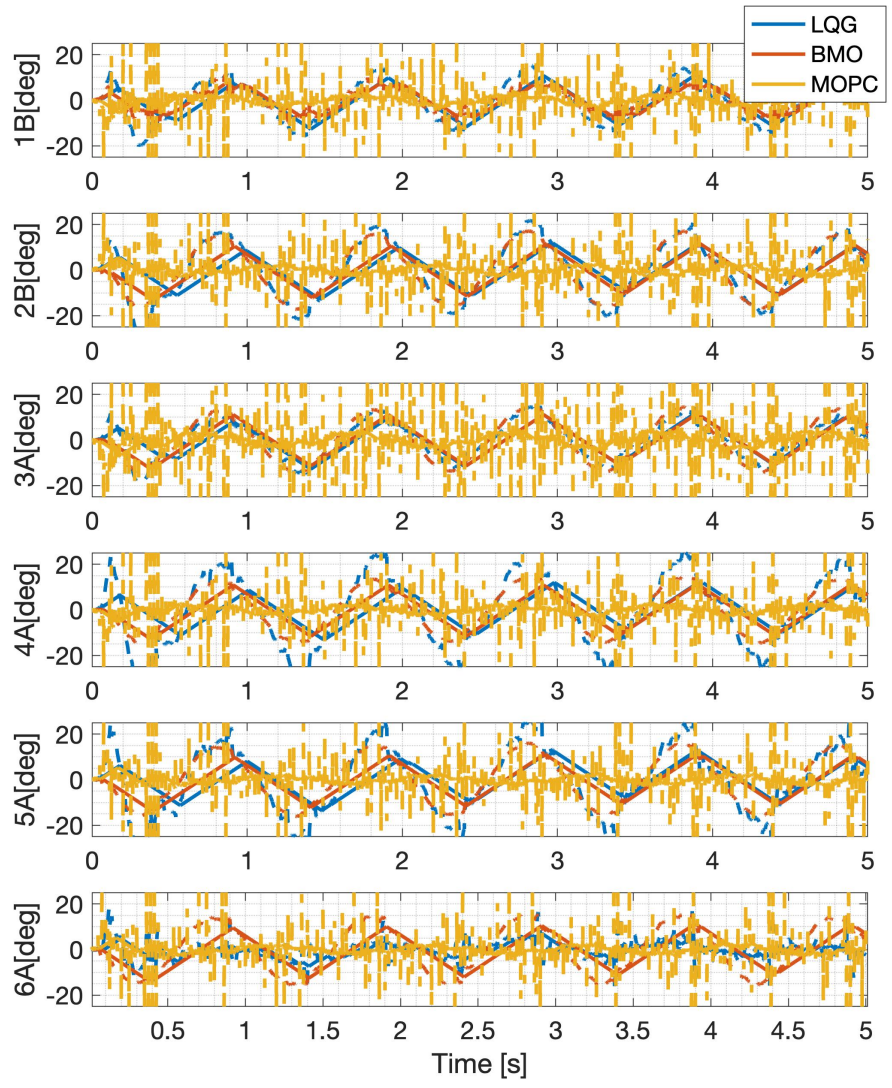

Fig. 15 Simulink simulation controller flap deflections

multi-objective control. This was demonstrated through analytical analysis, and verified using a high-fidelity Simulink model which captures sampling and control saturation effects. The Multi-Objective + Prediction Correction controller was also evaluated. This is a novel control strategy that attempts to correct for errors in predicting the gust disturbance. Despite not demonstrating a performance or robustness advantage in this context, further investigation is warranted, given its sound mathematical development. With alternate plant output and/or estimation techniques and tuning, this controller may be a valuable augmentation to the proven multi-objective control technique.

\section{Acknowledgments}

Funding for this work was provided by the Advanced Air Transport Technology Project of the NASA Aeronautics Research Mission Directorate as well as a Phase III SBIR award to Scientific Systems Company, Inc. and the University of Washington.

\section{Appendix A: Matrix Definitions}

Matrix definitions for the $u_{n}$-loop-closed dynamics are as follows:

$$
\begin{aligned}
\bar{A} & =A+B_{n} K_{n x} \\
\bar{B}_{i} & =B_{i}+B_{n} K_{n r} \\
\bar{E} & =E+B_{n} K_{n w} \\
\bar{\Lambda}_{n} & =B_{n} \Lambda_{n} .
\end{aligned}
$$




$$
\begin{aligned}
& \bar{M}_{x}=M_{x}+M_{u_{n}} K_{n x} \\
& \bar{M}_{r}=M_{u_{n}} K_{n r} \\
& \bar{M}_{w}=M_{w}+M_{u_{n}} K_{n w} \\
& \bar{\Lambda}_{M}=M_{u_{n}} \Lambda_{n} . \\
& \bar{C}_{D_{x}}=C_{D_{x}}+C_{D_{u_{n}}} K_{n x}+\Lambda_{n}^{T} C_{D_{x u_{n}}}^{T}+\Lambda_{n}^{T}\left(C_{D_{u^{2} n n}^{T}}^{T}+C_{D_{u^{2} n n}}\right) K_{n x} \\
& \bar{C}_{D_{r}}=C_{D_{u_{n}}} K_{n r}+\Lambda_{n}^{T}\left(C_{D_{u^{2} n n}^{T}}^{T}+C_{D_{u^{2} n n}}\right) K_{n r} \\
& \bar{C}_{D_{w}}=C_{D_{u_{n}}} K_{n w}+\Lambda_{n}^{T}\left(C_{D_{u^{2} n n}^{T}}^{T}+C_{D_{u^{2} n n}}\right) K_{n w} \\
& \bar{C}_{D_{u_{m}}}=C_{D_{u_{m}}}+\Lambda_{n}^{T}\left(C_{D_{u^{2} m n}}^{T}+C_{D_{u^{2} n m}}\right) \\
& \bar{\Lambda}_{C}=C_{D_{u_{n}}} \Lambda_{n}+\Lambda_{n}^{T} C_{D_{u^{2} n n}} \Lambda_{n} \\
& \bar{C}_{D_{x^{2}}}=C_{D_{x^{2}}}+C_{D_{x u n}} K_{n x}+K_{n x}^{T} C_{D_{u^{2} n n}} K_{n x} \\
& \bar{C}_{D_{u^{2} m m}}=C_{D_{u^{2} m m}} \\
& \bar{C}_{D_{x u m}}=C_{D_{x u m}}+K_{n x}^{T}\left(C_{D_{u^{2} m n}^{T}}^{T}+C_{D_{u^{2} n m}}\right) \\
& \bar{C}_{D_{\text {rum }}}=K_{n r}^{T}\left(C_{D_{u^{2} m n}^{T}}^{T}+C_{D_{u^{2} n m}}\right) \\
& \bar{C}_{D_{w u m}}=K_{n w}^{T}\left(C_{D_{u^{2} m n}^{T}}^{T}+C_{D_{u^{2} n m}}\right) \\
& \bar{C}_{D_{u^{2} c m}}=\left(C_{D_{u^{2} m c}^{T}}^{T}+C_{D_{u^{2} c m}}\right) \\
& \bar{C}_{D_{r x}}=K_{n r}^{T} C_{D_{x u_{n}}}^{T}+K_{n r}^{T}\left(C_{D_{u^{2} n n}^{T}}^{T}+C_{D_{u^{2} n n}}\right) K_{n x} \\
& \bar{C}_{D_{w x}}=K_{n w}^{T} C_{D_{x u n}}^{T}+K_{n w}^{T}\left(C_{D_{u^{2} n n}}^{T}+C_{D_{u^{2} n n}}\right) K_{n x} \\
& \bar{C}_{D_{r^{2}}}=K_{n r}^{T} C_{D_{u^{2} n n}} K_{n r} \\
& \bar{C}_{D_{r w}}=K_{n r}^{T}\left(C_{D_{u^{2} n n}^{T}}^{T}+C_{D_{u^{2} n n}}\right) K_{n w} \\
& \bar{C}_{D_{w^{2}}}=K_{n w}^{T} C_{D_{u^{2} n n}} K_{n w} .
\end{aligned}
$$

Matrix definitions for the $u_{m}$-loop-closed dynamics are given next.

$$
\begin{gathered}
\overline{\bar{A}}=\bar{A}+B_{m} K_{x} \\
\overline{\bar{B}}_{i}=\bar{B}_{i}+B_{m} K_{r} \\
\overline{\bar{E}}=\bar{E}+B_{m} K_{w} \\
\overline{\bar{\Lambda}}_{n}=\bar{\Lambda}_{n}+B_{m} \Lambda . \\
\overline{\bar{C}}=C+D_{n} K_{n x}+D_{m} K_{x} \\
\overline{\bar{D}_{i}=} D_{n} K_{n r}+D_{m} K_{r} \\
\overline{\bar{F}}=F+D_{n} K_{n w}+D_{m} K_{w} \\
\overline{\bar{\Lambda}}_{y}=D_{n} \Lambda_{n}+D_{m} \Lambda .
\end{gathered}
$$




\section{Appendix B: Gain Definitions}

The $u_{m}$ gains in Eq. (13) require several nested definitions. They are summarized in reverse implementation order here, where $W$ is the solution of the Riccati equation in Eq. 65.

$$
\begin{aligned}
& K_{x}=-\bar{R}^{-1}\left(\frac{1}{2} q_{D} \bar{C}_{D_{x u_{m}}}^{T}+q_{M} M_{u_{m}}^{T} \bar{M}_{x}+B_{m}^{T} W\right) \\
& K_{r}=-\bar{R}^{-1}\left(\frac{1}{2} q_{D} \bar{C}_{D_{r u_{m}}}^{T}+q_{M} M_{u_{m}}^{T} \bar{M}_{r}+B_{m}^{T} V_{r}\right) \\
& K_{w}=-\bar{R}^{-1}\left(\frac{1}{2} q_{D} \bar{C}_{D_{w u m}}^{T}+q_{M} M_{u_{m}}^{T} \bar{M}_{w}+B_{m}^{T} V_{w}\right) \\
& \Lambda=-\bar{R}^{-1}\left(\frac{1}{2} q_{D} \bar{C}_{D_{u_{m}}}^{T}+q_{M} M_{u_{m}}^{T} \bar{\Lambda}_{M}+B_{m}^{T} V_{0}\right) \\
& W \overline{\bar{A}}+\overline{\bar{A}}^{T} W-W B_{m} \bar{R}^{-1} B_{m} W+\overline{\bar{Q}}=0 \\
& V_{r}=-\overline{\bar{V}}^{-1}\left[W \bar{B}_{i}+\frac{1}{2} q_{D} \bar{C}_{D_{r x}}^{T}+q_{M} \bar{M}_{x}^{T} \bar{M}_{r}-W B_{m} \bar{R}^{-1}\left(\frac{1}{2} q_{D} \bar{C}_{D_{r u m}}^{T}+q_{M} M_{u_{m}}^{T} \bar{M}_{r}\right)\right. \\
& \left.-\left(\frac{1}{2} q_{D} \bar{C}_{D_{x u_{m}}}+q_{M} \bar{M}_{x}^{T} M_{u_{m}}\right) \bar{R}^{-1}\left(\frac{1}{2} q_{D} \bar{C}_{D_{r u_{m}}}^{T}+q_{M} M_{u_{m}}^{T} \bar{M}_{r}\right)\right] \\
& V_{w}=-\overline{\bar{V}}^{-1}\left[W \bar{E}+\frac{1}{2} q_{D} \bar{C}_{D_{w x}}^{T}+q_{M} \bar{M}_{x}^{T} \bar{M}_{w}-W B_{m} \bar{R}^{-1}\left(\frac{1}{2} q_{D} \bar{C}_{D_{w u m}}^{T}+q_{M} M_{u_{m}}^{T} \bar{M}_{w}\right)\right. \\
& \left.-\left(\frac{1}{2} q_{D} \bar{C}_{D_{x u_{m}}}+q_{M} \bar{M}_{x}^{T} M_{u_{m}}\right) \bar{R}^{-1}\left(\frac{1}{2} q_{D} \bar{C}_{D_{w u_{m}}}^{T}+q_{M} M_{u_{m}}^{T} \bar{M}_{w}\right)\right] \\
& V_{0}=-\overline{\bar{V}}^{-1}\left[W \bar{\Lambda}_{n}+\frac{1}{2} q_{D} \bar{C}_{D_{x}}^{T}+q_{M} \bar{M}_{x}^{T} \bar{\Lambda}_{M}-W B_{m} \bar{R}^{-1}\left(\frac{1}{2} q_{D} \bar{C}_{D_{u_{m}}}^{T}+q_{M} M_{u_{m}}^{T} \bar{\Lambda}_{M}\right)\right. \\
& \left.-\left(\frac{1}{2} q_{D} \bar{C}_{D_{x u_{m}}}+q_{M} \bar{M}_{x}^{T} M_{u_{m}}\right) \bar{R}^{-1}\left(\frac{1}{2} q_{D} \bar{C}_{D_{u_{m}}}^{T}+q_{M} M_{u_{m}}^{T} \bar{\Lambda}_{M}\right)\right] \\
& \overline{\bar{V}}=\overline{\bar{A}}^{T}-W B_{m} \bar{R}^{-1} B_{m} \\
& \overline{\bar{A}}=\bar{A}-\frac{1}{2} q_{D} B_{m} \bar{R}^{-1} \bar{C}_{D_{x u_{m}}}^{T}-q_{M} B_{m} \bar{R}^{-1} M_{u_{m}}^{T} \bar{M}_{x} \\
& \overline{\bar{Q}}=Q+q_{D} \bar{C}_{D_{x^{2}}}+q_{M} \bar{M}_{x}^{T} \bar{M}_{x}-\left(\frac{1}{2} q_{D} \bar{C}_{D_{x u_{m}}}+q_{M} \bar{M}_{x}^{T} M_{u_{m}}\right) \bar{R}^{-1}\left(\frac{1}{2} q_{D} \bar{C}_{D_{x u_{m}}}^{T}+q_{M} M_{u_{m}}^{T} \bar{M}_{x}\right) \\
& \bar{R}=R+q_{D} \bar{C}_{D_{u^{2} m m}}+q_{M} M_{u_{m}}^{T} M_{u_{m}}
\end{aligned}
$$

The $u_{c}$ gains in Eq. 21] are provided next.

$$
\begin{gathered}
\bar{K}_{e}=-\bar{R}_{c}^{-1}\left(\Gamma^{T}+B_{m}^{T} P_{c}\right) \\
\bar{K}_{w}=-\bar{R}_{c}^{-1} B_{m}^{T} V_{c w} \\
\Gamma=q_{c M} M_{x}^{T} M_{u_{m}}+q_{c D} C_{D_{x}}^{T} C_{D_{u_{m}}} \\
\bar{Q}_{c}+P_{c} A_{c}+A_{c}^{T} P_{c}-P_{c} B_{m} \bar{R}_{c}^{-1} B_{m}^{T} P_{c}=0 \\
V_{c w}=-\left(A_{c}^{T}-P_{c} B_{m} \bar{R}_{c}^{-1} B_{m}^{T}\right)^{-1} P_{c} \overline{\bar{E}} \\
A_{c}=\overline{\bar{A}}-B_{m} \bar{R}_{c}^{-1} \Gamma^{T} \\
\bar{Q}_{c}=Q_{c}+q_{c M} M_{x}^{T} M_{x}+q_{c D} C_{D_{x}}^{T} C_{D_{x}}-\Gamma \bar{R}_{c}^{-1} \Gamma^{T} \\
\bar{R}_{c}=R_{c}+q_{c M} M_{u_{m}}^{T} M_{u_{m}}+q_{c D} C_{D_{u_{m}}}^{T} C_{D_{u_{m}}}
\end{gathered}
$$




\section{References}

[1] Nguyen, N. et al., "Elastically shaped wing optimization and aircraft concept for improved cruise efficiency," 51 ${ }^{\text {st }}$ AIAA Aerospace Sciences Meeting Including the New Horizons Forum and Aerospace Exposition, 2013, p. 141.

[2] Ferrier,Y., Nguyen, N., and Ting, E., "Real-Time Adaptive Least-Squares Drag Minimization for Performance Adaptive Aeroelastic Wing," 34 ${ }^{\text {th }}$ AIAA Applied Aerodynamics Conference, Washington D.C. June 2016.

[3] Nguyen, N. et al., "Experimental Investigation of a Flexible Wing with a Variable Camber Continuous Trailing Edge Flap Design," 32 $2^{\text {nd }}$ AIAA Applied Aerodynamics Conference, Atlanta, GA, June 2014.

[4] Precup, N., Mor, M., and Livne, E., "Design, Construction, and Tests of an Aeroelastic Wind Tunnel Model of a Variable Camber Continuous Trailing Edge Flap (VCCTEF) Concept Wing," 32 ${ }^{\text {nd }}$ AIAA Applied Aerodynamics Conference, Atlanta, GA, June 2014.

[5] Nguyen, N. et al., "Wind Tunnel Investigation of a Flexible Wing High-Lift Configuration with a Variable Camber Continuous Trailing Edge Flap Design," 33 ${ }^{\text {rd }}$ AIAA Applied Aerodynamics Conference, Dallas, TX, June 2015.

[6] Nguyen, N. et al., "Real-Time Adaptive Drag Minimization Wind Tunnel Investigation of a Flexible Wing with Variable Camber Continuous Trailing Edge Flap System," 37 ${ }^{\text {rd }}$ AIAA Applied Aerodynamics Conference, Dallas, TX, June 2019.

[7] Rivers, M.B. and Dittberner, A, "Experimental Investigations of the NASA Common Research Model," Journal of Aircraft, Vol. 51, No. 4, July 2014, pp. 1183-1193.

[8] Cramer, N. and Nguyen, N., "Development of an Aeroservoelastic Model for Gust Load Alleviation of the NASA Common Research Model Wind Tunnel Experiment," AIAA SciTech 2020 Forum, Orlando, FL, January 2020.

[9] Hashemi, K.E., Nguyen, N., and Drew, M., "Time-Varying Weights in Multi-Objective Optimal Control for Flexible Wing Aircraft," AIAA SciTech 2019 Forum, San Diego, CA, January 2019.

[10] Drew, M. et al., "Multi-objective Optimal Control of the 6-DoF Aeroservoelastic Common Research Model with Aspect Ratio 13.5 Wing," AIAA SciTech 2019 Forum, San Diego, CA, January 2019.

[11] Hashemi, K.E. et al., "Performance Optimizing Gust Load Alleviation Control of Flexible Wing Aircraft," AIAA SciTech 2018 Forum, Kissimmee, FL, January 2018.

[12] Xiong, J. et al., "Computation of Flow Induced by Gust Generator in a Wind-Tunnel," 37 ${ }^{\text {rd }}$ AIAA Applied Aerodynamics Conference, Dallas, TX, June 2019.

[13] Lavretsky, E. and Wise, K. Robust and Adaptive Control: With Aerospace Applications, Advanced Textbooks in Control and Signal Processing, Springer London, 2012. 